\title{
Satisfacción de los Adultos Mayores por el Uso de Audífonos otorgados por el Programa Chileno de Garantías Explícitas en Salud (GES)
}

\author{
Miguel A. Bustamante Ubilla ${ }^{(1)}$, Cristian L. Vidal Silva ${ }^{(2)}$ y Leopoldo P. López ${ }^{(3)}$ \\ (1) Escuela de Ingeniería Comercial, Facultad de Economía y Negocios, Universidad de Talca, \\ Campus Lircay, Avenida Lircay S/N, Talca-Chile (e-mail: mabu@utalca.cl) \\ (2) Departamento de Computación e Informática, Facultad de Ingeniería, Universidad de Playa Ancha, \\ Avenida Leopoldo Carvallo 270, Playa Ancha, Valparaíso-Chile (e-mail: cristian.vidal@upla.cl) \\ (3) Instituto de Investigación y Desarrollo Educacional, IIDE, Universidad de Talca Campus Lircay, \\ Avenida Lircay S/N, Talca-Chile (e-mail: Ilopez@utalca.cl)
}

Recibido Abr. 24, 2014; Aceptado Jun. 26, 2014; Versión final recibida Ago. 26, 2014

\begin{abstract}
Resumen
Este trabajo describe un estudio acerca de la satisfacción usuaria de adultos mayores en Chile por el uso de audífonos otorgados por el programa Garantías Explícitas en Salud en Chile (GES). Para ello se analiza las necesidades para el uso de audífonos en una muestra representativa de adultos mayores en Chile (población objetivo) y se discute sobre los beneficios logrados por el uso de audífonos en la población objetivo. Además, este trabajo presenta un análisis de factores de componentes principales de un estudio de percepción de calidad de vida por el uso de audífonos en adultos mayores. Se concluye que hay relación directa entre el uso de audífonos y la calidad de vida de adultos mayores en Chile que los usan.
\end{abstract}

Palabras clave: adulto mayor, audífonos, satisfacción, calidad de vida, GES

\section{Satisfaction of Senior People for Using Earphones given by the Chilean Program Explicit Health Guaranties (GES)}

\begin{abstract}
This paper describes a study about the user satisfaction of senior people in Chile for the use of earphones given by the Chilean Program Explicit Health Guaranties (GES). For this reason, the requirements established for the use of earphones in a representative sample of older people in Chile (target population) are analyzed and the benefits achieved by the use of earphones in the target population are discussed. In addition, this paper presents an analysis of factors of the main components of life quality perception by the use of earphones in senior people. It is concluded that there is direct relation between the use of earphones and quality life of senior people that used them.
\end{abstract}

Keywords: senior people, earphones, user satisfaction, life quality, GES 


\section{INTRODUCCIÓN}

La relación "responsabilidad social y salud" y las significativas causalidades que existen entre los temas "sociales y económicos" con los temas de "salud" y la "calidad de vida" (Salvador, 2007), permiten validar la denominada "dimensión ética en salud" (Fanden et al., 2013; Norman et al., 2011) ya que estos temas contribuyen a crear "condiciones de calidad de vida" superiores (Garrafa, 2005). Las relaciones conceptuales y empíricas entre responsabilidad social y calidad de vida (Fernández y Gutiérrez, 2013) impactan los procesos (Maani et al., 1994) y potencia la innovación (Flynnet, 1994), y son fuente de satisfacción en la sociedad (Larson y Sinha, 1995) y potencian una "Responsabilidad Social Empresarial" (RSE) (Adam, 1994; Keen y Birley, 1995.; Kessler, 1995.; Nilsson et al., 2001).

En este escenario, se ha producido un consenso general desde la década de los 80 (Deming, 1986; Ishikawa, 1986; Juran y Tryna, 1994; Summers; 2006. Feigenbaum 2010) respecto a un paradigma de calidad de vida y que, en servicios de salud fueron anunciados por Avedis Donabedian a finales de esa década (Donabedian, 1988). De esta manera se fundamenta el presente estudio en el imperativo de la responsabilidad social derivada de la calidad de vida y donde uno de los instrumentos más conocidos para medirla es la escala de satisfacción con la vida (Diener et al., 1985) ratificada por diversos autores al concordar que la satisfacción puede ser entendida como la suma de la satisfacción en diferentes esferas o dominios que la componen (Andrews y Withey, 1976; Campbell et al., 1976; Diener, 1984; Day, 1987; Hsieh, 2003).

La presente investigación, un complemento del trabajo de Bustamante et al. (2014) que trata el impacto del uso de audífonos en adultos mayores en Chile, se concentra en la percepción del bienestar subjetivo (BS) (Diener, 1984; Diener et al., 1999, Diener y Lucas, 2000) de la satisfacción personal de los adultos mayores por el uso de audífonos, analizando los componentes "desempeño auditivo subjetivo y satisfacción en adultos mayores" (Diener, 1984; Diener et al., 1985) para describir algunas de las condiciones de vida de este grupo etario, haciendo coincidir componentes subjetivos y objetivos en esta dimensión particular de la calidad de vida (Castellón y Sánchez del Pino, 2001). El propósito de este estudio es determinar los principales cambios en la calidad de vida de los adultos mayores que han accedido a la entrega de audífono por beneficio GES. Para ello, se trabaja sobre una muestra significativa de la población en una comuna de Chile, esto es, Talca, en la comuna de Talca entre los años 2008 - 2011, con la meta de determinar si la entrega de audífono ha implicado cambios en su calidad de vida e indicar cuál es la causa de no utilización de audífono, para finalmente, establecer propuestas en beneficio en la calidad de vida de los adultos mayores.

\section{METODOLOGÍA}

Para realizar este estudio, se hizo una revisión exhaustiva de la Guía Clínica MINSAL N 56, del año 2007 (Minsal, 2013) correspondiente a la Patología "hipoacusia bilateral en personas de 65 años y más que requieren audífonos". Como fuentes de información secundaria, se recolectó datos desde la oficina GES del Hospital Regional de Talca y de una organización externa adjudicataria para la entrega de audífonos en este periodo (Auditron, 2013), la que reportó un número de 50 datos de adultos mayores sujeto de la entrega y se obtuvo el registro de números telefónicos y direcciones de quienes se encuentran formalmente incorporados al sistema oficial de entrega de Audífonos GES por parte de esta organización encomendada por FONASA.

Se planificaron entrevistas directas con el instrumento prediseñado considerando como universo a los adultos mayores que han recibido el beneficio GES de entrega de audífonos con prestador FONASA y se consideró como muestra aquellos que tienen más de un año desde la entrega del beneficio periodo comprendido desde agosto del 2008 y hasta el año 2011. Se realizó el diseño de un cuestionario combinado de recogida de datos, conformado por el "Cuestionario Internacional de Resultado de Uso de Audífonos (CIRUA" seguido de un "Cuestionario de Percepción de Calidad de Vida" mediante la modificación parcial por asimilación al instrumento de satisfacción y calidad de vida (Shortened Hearing Handicap Inventory y for the Elderly, HHIES). El instrumento diseñado fue sometido a diversas validaciones a través de consultas a expertos y piloteo mediante su aplicación a un grupo control a fin de corregir eventuales errores de diseño y asegurar su comprensión. Finalmente fue aplicado, previa validación y correcciones de acuerdo con las observaciones recogidas.

\section{ANÁLISIS DE DATOS}

Se procedió al análisis socio-demográfico de la muestra sobre la base de los datos recogidos en las entidades informantes, Hospital Regional de Talca y Organización adjudicataria para la distribución de los Audífonos a los Adultos mayores debidamente registrados. 


\section{Estadística Descriptiva}

Se elaboró una síntesis estadística de los resultados de la aplicación del "Cuestionario Internacional de Resultado de Uso de Audífonos" (CIRUA). La fase inicial del estudio se aplicó sobre la base de una escala Likert de 5 puntos crecientes (Likert, 1932). Además, se elaboró una síntesis de la aplicación del "Cuestionario Percepción De Calidad De Vida", diseñada sobre la base de una Escala discreta de 3 categorías de respuesta, a decir: Sí, A Veces y No (Delgado y Gutiérrez, 1994). Ambas síntesis estadísticas consideraron la verificación de los registros Válidos y Perdidos, así como las cifras de población Urbana, Rural y Total, respecto de las cuales se determinó las cifras del Sistema, las respectivas Frecuencias y Porcentajes Válidos y Acumulados, para finalmente determinar los estadísticos descriptivos Media, Mediana y Moda. Complementariamente se realizó un análisis de las respuestas recogidas de la Pregunta Abierta incorporada al Cuestionario de Calidad de Vida.

\section{Estadística Inferencial}

Se realizó un "Análisis de Factores de Componentes Principales" de los datos (Ferrando, 1994; García et al., 2000), por cuanto dicho método permite el análisis de una gran cantidad de datos agrupándolos en Factores constituidos por componentes principales validados por cargas estadísticas representativas. Aunque los datos son aún escasos en número, su aplicación fue posible en esta fase de la investigación por cuanto es compatible con las diversas escalas previstas en los diversos métodos que hacen factible su aplicación, a decir, la "Escala de Bienestar Psicológico" (EBP), que trabaja con 13 ítems y con tres opciones de respuesta (de "En desacuerdo" 1, a "De acuerdo" 3). La Escala Bienestar Subjetivo, que se aplica a través de una escala tipo Likert (Likert, 1932), en este caso con cinco opciones de respuesta. Además, se aplica la EBP de Ryff (1989) que utiliza la secuencia 1 (completamente en desacuerdo) a 6 (completamente de acuerdo). Por otra parte, la OHQ (Oxford Happyness Questionaire) (Diener et al., 2000) que también aplica una escala de 1 a 6 , finalmente, la Escala de Felicidad Subjetiva, EFS, contempla, así mismo, una escala Likert de 1 a 7 puntos (Likert, 1932).

\section{Análisis de la necesidad de uso y beneficios logrados por el uso de audífonos}

En general se hicieron dos preguntas a los adultos mayores, la primera, el por qué quiso ponerse audífonos $y$, la segunda, qué cosas han cambiado en su vida por su utilización. Al respecto, las tablas siguientes resumen las respuestas recogidas relacionando las respuestas con los datos demográficos que definen la muestra y que permiten caracterizar y sintetizar algunas conclusiones. Tabla 1 y tabla 2 muestran los resultados asociados a estas interrogantes. En general se confirme que la necesidad de utilizar audífonos resulta del déficit auditivo diagnosticado de adultos mayores dependientes, seguido de la avanzada edad de dichas personas (75-85 años) que, en general, residen en las zonas urbanas y son de sexo femenino.

Entre las razones que explican la necesidad del uso de los audífonos así como los aspectos de la vida que han cambiado entre los usuarios de esta tecnología se menciona la necesidad de sociabilizar, es decir, de mejorar sus opciones de integración social lo que en sí justifica el cómo, cuándo y por qué el Sector Salud ha priorizado la hipoacusia bilateral como una problema de salud que merece la entregar de un beneficio que favorezca, en sí mismo, la calidad de vida de los adultos mayores. Los adultos mayores manifiestan que, efectivamente ellos utilizan sus audífonos de manera regular, al tiempo que, también algunos de ellos, en escaso porcentaje, justifican el no utilizar sus respectivos audífonos en una decisión personal así como quienes señalan no haber percibido un cambio significativo al utilizarlos o porque debieron enfrentar diversas dificultades técnicas, en suma, todas razones que explican el por qué simplemente lo dejaban de usar.

Tabla 1: Por qué se quiso poner audífonos según actividad-años cumplidos-residencia-sexo. Nec. Aud. = Necesidad Auditiva / Nec. Soc. = Necesidad De Sociabilizar / Dep.: Vive con algún familiar / Jub.: Jubilado / F: Femenino / M: Masculino

\begin{tabular}{|c|c|c|c|c|c|c|c|c|c|c|c|c|c|c|}
\hline & \multicolumn{3}{|c|}{ Actividad } & \multirow[b]{2}{*}{ Total } & \multicolumn{3}{|c|}{ Años Cumplidos } & \multirow[b]{2}{*}{ Total } & \multicolumn{2}{|c|}{ Residencia } & \multirow[b]{2}{*}{ Total } & \multicolumn{2}{|c|}{ Sexo } & \multirow[b]{2}{*}{ Total } \\
\hline & Activo & Dep. & Jub. & & $\begin{array}{l}65- \\
75\end{array}$ & $\begin{array}{c}75- \\
85\end{array}$ & $\begin{array}{l}85- \\
\text { Más }\end{array}$ & & Urbana & Rural & & $\mathrm{F}$ & M & \\
\hline $\begin{array}{l}\text { Nec. } \\
\text { Aud. }\end{array}$ & 7 & 15 & 3 & 25 & 9 & 12 & 4 & 25 & 23 & 3 & 26 & 17 & 9 & 26 \\
\hline $\begin{array}{l}\text { Nec. } \\
\text { Soc. }\end{array}$ & 1 & 3 & 0 & 4 & 1 & 2 & 1 & 4 & 3 & 0 & 3 & 4 & 0 & 4 \\
\hline Total & 8 & 18 & 3 & 29 & 10 & 14 & 5 & 29 & 26 & 3 & 29 & 21 & 9 & 30 \\
\hline
\end{tabular}


Tabla 2: Asuntos que han cambiado desde que tiene su audífono según actividad, sexo, residencia, edad. Dep.: Vive con algún familiar / Jub: Jubilado / F: Femenino / M: Masculino

\begin{tabular}{|c|c|c|c|c|c|c|c|c|c|c|c|c|c|c|}
\hline & \multicolumn{3}{|c|}{ Actividad } & \multirow[b]{2}{*}{ Total } & \multicolumn{2}{|c|}{ Residencia } & \multirow[b]{2}{*}{ Total } & \multicolumn{2}{|c|}{ Sexo } & \multirow[b]{2}{*}{ Total } & \multicolumn{3}{|c|}{ Años Cumplidos } & \multirow[b]{2}{*}{ Total } \\
\hline & Activo & Dep. & Jub. & & Urb & Rural & & $\mathrm{F}$ & M & & $\begin{array}{l}65- \\
75\end{array}$ & $\begin{array}{c}75- \\
85\end{array}$ & $\begin{array}{l}85- \\
\text { Más }\end{array}$ & \\
\hline Ninguna & 0 & 4 & 0 & 4 & 3 & 1 & 4 & 3 & 1 & 4 & 0 & 0 & 3 & 3 \\
\hline $\begin{array}{l}\text { Realizar } \\
\text { Actividades }\end{array}$ & 2 & 3 & 1 & 6 & 5 & 1 & 6 & 4 & 2 & 6 & 4 & 2 & 0 & 6 \\
\hline $\begin{array}{l}\text { Interacción } \\
\text { Social }\end{array}$ & 6 & 12 & 2 & 20 & 19 & 1 & 20 & 15 & 6 & 21 & 6 & 12 & 2 & 20 \\
\hline Total & 8 & 19 & 3 & 30 & 27 & 3 & 30 & 22 & 9 & 31 & 10 & 1 & 5 & 29 \\
\hline
\end{tabular}

Análisis de factores de percepción de calidad de vida por el uso de audífonos

Para los efectos de analizar en profundidad las percepciones de satisfacción usuaria y calidad de vida de los adultos mayores por el uso de audífonos, se procedió a la determinación de los Factores de Componentes Principales del Cuestionario de Percepción de Calidad de Vida. La tabla 4 detalla los resultados del análisis estadístico (SPSS) de Análisis de Factores de Componentes rotados.

Tabla 4: Factores de componentes principales del cuestionario de calidad de vida.

\begin{tabular}{|c|c|c|}
\hline & Factor 1 "Mejor salud para la vida diaria" & \\
\hline $\mathrm{N}^{\circ}$ & Componentes Cuestionario Percepción de Calidad de Vida & Cargas \\
\hline 1 & A cambiado su vida con el uso de audífono & 0,734 \\
\hline 5 & Puede escuchar bien radio y televisión & 0,848 \\
\hline \multirow[t]{4}{*}{11} & Ha mejorado su salud con la entrega de audífonos & 0,806 \\
\hline & Varianza Total Explicada (Fi) & 21,183 \\
\hline & Varianza Acumulada $\sum(\mathrm{Fi})$ & 21,183 \\
\hline & Factor 2 "Diferente pero no inválido" & \\
\hline $\mathrm{N}^{\circ}$ & Componentes Cuestionario Percepción Calidad de Vida & Cargas \\
\hline 10 & Se siente diferente de sus pares al tener utilizar audífonos & 0,827 \\
\hline \multirow[t]{4}{*}{15} & Se siente invalido por tener que utilizar audífono & 0,913 \\
\hline & Varianza Total Explicada (Fi) & 16,418 \\
\hline & Varianza Acumulada $\sum(\mathrm{Fi})$ & 37,601 \\
\hline & Factor 3 "Una mejor vida diaria sin caídas" & \\
\hline $\mathrm{N}^{\circ}$ & Componentes Cuestionario Percepción Calidad de Vida & Cargas \\
\hline 2 & Ha ayudado en sus tareas diarias el uso de audífono & 0,506 \\
\hline \multirow[t]{4}{*}{3} & Ha tenido caídas desde la entrega del audífono & 0,825 \\
\hline & Varianza Total Explicada (Fi) & 11,719 \\
\hline & Varianza Acumulada $\sum(\mathrm{Fi})$ & 49,320 \\
\hline & Factor 4 "Familia más cercana y mejor salud" & \\
\hline $\mathrm{N}^{\circ}$ & Componentes Cuestionario Percepción Calidad de Vida & Cargas \\
\hline 6 & Su familia se aproxima más a usted desde la entrega del audífono & 0,851 \\
\hline \multirow[t]{4}{*}{12} & Siente mareos posterior a la entrega de audífonos & 0,600 \\
\hline & Varianza Total Explicada (Fi) & 9,680 \\
\hline & Varianza Acumulada $\Sigma(\mathrm{Fi})$ & 59,000 \\
\hline & Factor 5 "Uso el audífono a pesar de los ruidos" & \\
\hline $\mathrm{N}^{\circ}$ & Componentes Cuestionario Percepción Calidad de Vida & Cargas \\
\hline 4 & El audífono genera ruidos que le molesten & 0,822 \\
\hline \multirow[t]{4}{*}{9} & El ruido que genera el audífono lo hace no usarlo & 0,830 \\
\hline & Varianza Total Explicada (Fi) & 8,681 \\
\hline & Varianza Acumulada $\sum(\mathrm{Fi})$ & 67,680 \\
\hline & Factor 6 "Los audifonos me han sido útiles" & \\
\hline $\mathrm{N}^{\circ}$ & Componentes Cuestionario Percepción Calidad de Vida & Cargas \\
\hline \multirow[t]{3}{*}{8} & Siente que la entrega de audífonos ha sido inútil & 0,901 \\
\hline & Varianza Total Explicada (Fi) & 6,972 \\
\hline & Varianza Acumulada $\Sigma(\mathrm{Fi})$ & 74,653 \\
\hline
\end{tabular}


La Tabla 4 detalla el análisis de factores del cuestionario de calidad de vida y de la matriz de componentes rotados(a), se determinaron 6 Factores que focalizan el análisis de las respuestas recogidas. Además, se describen los 6 Factores determinados con sus títulos descriptores y que en conjunto explican el $74,65 \%$ de la varianza total, señalado para cada factor los componentes principales con sus respectivas cargas.

El Factor 1 denominado "Mejor salud para la vida diaria", pone en evidencia cómo ha cambiado la vida de los adultos mayores con el uso de audífono, medio que les ha permitido escuchar bien radio y la televisión mejorando su salud y con ello su calidad de vida. Este factor explica el $21,18 \%$ de la varianza total del estudio. El Factor 2, "Diferente pero no inválido", explicando el $16,42 \%$ pone en evidencia que, efectivamente los adultos mayores se sienten diferentes ante la vista de sus respectivos pares al tener utilizar audífonos, reconociendo una condición diferencial que aceptan pero que sin embargo no les hace sentirse inválidos frente a los demás. Por lo que se recogió de las respuestas abiertas, los adultos mayores asumen su estado llevando con dignidad y satisfacción la necesidad de utilizar audífonos para escuchar.

Respecto del Factor 3, que explica un $11,72 \%$ de la varianza total y que denomina "Una mejor vida diaria sin caídas", pone en evidencia que los audífonos les han ayudado en sus tareas diarias haciendo que su vida cotidiana sea más placentera y al mismo tiempo les ha contribuido a evitar las caídas, por cuanto confirman sin lugar a dudas que éstas se han reducido desde la recepción de sus respectivos audífonos.

El Factor 4, titulado "Familia más cercana y mejor salud", explicando el 9,7\% de la varianza total, hace mención a la importancia de la familia en una vida con calidad. Manifiestan que efectivamente ésta se a mejorado por cuanto sus respectivas familias se han aproximado más a ellos desde la recepción de sus audífonos. Agregan además que los beneficios se han extendido hacia los aspectos clave de la salud dado que, desde la entrega que se les hizo de los audífonos, ya no han sentido los mareos que al perecer antes les eran habituales.

Reconociendo que lo tecnológico evidentemente no es el fuerte de loa adultos mayores, el Factor 5 titulado "Uso el audífono a pesar de los ruidos" y, explicando el $8,68 \%$ de la varianza total, ratifica el hecho que los audífonos recibidos por las personas generan ruidos molestos al ser usados, sin embargo, a pesar de estas dificultades, manifiestan que a pesar de ello, el ruido que generan los audífonos no les hace no usarlo, por el contrario, a pesar de las dificultades el beneficio de usarlos es valorado como superior a esas dificultades. Finalmente, el Factor 6, denominado "Los audífonos me han sido útiles", ratifica lo señalado por el Factor 5, en tanto señalan que, a pesar de las dificultades, los audífonos han sido útiles para los adultos mayores, explicando el $6,97 \%$ de la varianza total.

\section{CONCLUSIONES}

Desde la perspectiva del uso, los resultados obtenidos ratifican la relevancia de las condiciones de salud previas de los adultos mayores que han recibido audífonos, puesto que, al relacionar las alteraciones sensoriales con las característica socio-demográficas de la población estudiada, se ratifica el hecho que, según las percepciones recogidas, las condiciones previas de salud parecen haber sido superadas con el uso de los audífonos al no haber tenido caídas ni presentado mareos luego de haber recibido sus audífonos.

En general, los problemas asociados a la utilización de los audífonos tienen más que ver con la adecuación de las personas a la tecnología, por los problemas de acoplamiento que requieren de dispositivos especiales para solucionarlos o por eventuales problemas de diseño, molde y ajuste auricular así como por la complejidad de las instrucciones de colocación del dispositivo en la persona.

Finalmente, las percepciones de utilidad y satisfacción recogidas en el presente estudio por el uso de audífonos por parte de loa adultos mayores, permiten confirmar lo que señala la literatura, en el sentido que, el hecho de no escuchar provoca aislamiento social y por lo tanto soledad, todo lo cual puede derivar en efectos sobre el ánimo y gatillar efectos adversos que muchas veces se traduce en depresión. Concordante con lo anterior, los adultos mayores, a pesar de las diversas dificultades que enfrentan, por los ruidos que genera el uso de sus respectivos audífonos o por sentirse diferentes respecto de sus respectivos pares, valoran positivamente los beneficios que les reporta el uso de los audífonos.

En definitiva, los adultos mayores, valoran y aprecian en su real dimensión el haber recibido sus respectivos audífonos, reconocen la utilidad que les ha transferido a sus personas este medio de apoyo auricular y aprecian cómo les ha cambiado la vida y sus rutinas habituales, han podido volver a escuchas radio y televisión, se han reducido las caídas y reconocen estar viviendo la satisfacción de usar sus audífonos alcanzando un nivel de calidad de vida superior. 


\section{REFERENCIAS}

Adam, JR., Alternativas de Mejora y Rendimiento Organizacional y de las Prácticas de Calidad, Diario de Dirección de Operaciones, 12, 188-204 (1994).

Andrews, F. M. y Withey, S. B., Social Indicators of well-Being: Americans' Perceptions of Life Quality, Plenum, New York, USA (1976).

Auditrón, Estamos para Escuchar. http://www.auditron.cl/. Acceso: 12 de Noviembre (2013).

Bustamante, M., Vidal Silva, C. y López, L., Impacto del Uso de Audífonos para Adultos Mayores en Chile, Información Tecnológica, 25(3), 177-184 (2014).

Castellón, A. y Sanchez del Pino, M. A., Calidad de Vida y Salud, Revista MULT Gerontología, 11(1), 12-9 (2001).

Day, R. L., Relationships between Life Satisfaction and Consumer Satisfaction, in Marketing and the Qualityof-Life Interface, A. C. Samli, Westport, CT: Quorum Books, 289-311 (1987)

Delgado, J. M. y Gutiérrez, J., Métodos y Técnicas Cualitativas de Investigación en Ciencias Sociales, Síntesis, Madrid, España (1994).

Deming, W. E., Out of the Crisis, MIT Press, Massachusetts, USA (1986)

Diener, E., Subjective well-Being. Psychological Bulletin, 95, 542-575 (1984).

Diener, E., Emmons, R. A., Larsen, R. J. y Griffin, S., The Satisfaction with Life Scale, Journal of Personality Assessment, 49, 71-75 (1985).

Diener, E., Suh, E., Lucas, R. y Smith, H., Subjetive well-Being: Three Decades of Progress, Psychological Bulletin, 125 (2), 276-302 (1999).

Diener, E. y Lucas R., Explaining Differences in Societal Levels of Happiness: Relative Standards, Need Fulfillment, Culture, and Evaluation Theory, Journal of Happiness Studies: An Interdisciplinary Periodical on Subjective Well-Being, 1, 41-78 (2000).

Donabedian, A., Los Espacios de la Salud: Aspectos Fundamentales de la Organización de la Atención Médica, Fondo de Cultura Económica, México (1988).

Faden, R. R., Kass, N. E., Goodman, S. N., Pronovost, P., Tunis, S. y Beauchamp T. L., An Ethics Framework for a Learning Health Care System: A Departure from Traditional Research Ethics and Clinical Ethics, Hastings Center Report, 43(s1), s16-s27, Enero-Febrero (2013).

Feigenbaum, A. V., Total quality control: achieving productivity, market penetration, and advantage in the global economy, McGraw-Hill (2010).

Fernández, L. y Gutiérrez, M., Bienestar Social, Económico y Ambiental para las Presentes y Futuras Generaciones, Información Tecnológica, 24(2), 121-130 (2013).

Ferrando, P., Introducción al Análisis Factorial, PPU, 1era edición, Barcelona, España (1994).

Flynnet, B., Product Innovation, Revista An empirical study, Quality Management, 3, 56-90 (1994).

García, E., Flores, J. y Rodríguez, G., Análisis Factorial, Editorial La Muralla, (2000).

Garrafa, V., Inclusão Social no Contexto Político da Bioética, Revista Brasileira de Bioética, 1(2), 122-132 (2005).

Hsieh, C. M., Counting Importance: The Case of Life Satisfaction and Relative Domain Importance, Social Indicators Research, 68, 163-174 (2003).

Ishikawa, K., ¿Qué es el Control Total de la Calidad?: Modalidad Japonesa, Grupo Editorial Norma, Bogotá, Colombia (1986). 
Juran, J. M. y Tryna, F. M., Análisis y Planeación de la Calidad, Mc Graw Hill, USA (1994).

Keen, P. y Birley, B., Las Mejores Prácticas y el Despliegue de Políticas de Calidad, Simposio sobre Desarrollo de la Calidad, 38-42, Australia, Julio (1995).

Kessler, S., Atar la Satisfacción del Consumidor a la Planificación Estratégica, Congreso Anual de la Calidad, Cincinnati, USA, Mayo (1995).

Larson, P. y Sinha, A., Un Estudio de Directores de Calidad, Diario de Gestión de Calidad, 2, 53-66 (1995).

Likert, R., A Technique for the Measurement of Attitudes, Archives of Psychology, 140,1-55 (1932).

Maani, K., Putterill, M. y Sluti, D., El Análisis Empírico de la Calidad Mejoras en la Fabricación, Revista Internacional de Calidad y Confiabilidad, Gestión 1, 19-37 (1994).

Nilsson, L., Johnson, M. y Gustafsson, A., El Impacto de las Prácticas de Calidad en Satisfacción del Cliente y los Resultados Empresariales frente a las Organizaciones de Servicios del Producto, Diario de Gestión de la Calidad, 6, 5-27 (2001).

Norman, I. D., Aikins, M. K. y Binka, F. N., Ethics and Electronic Health Information Technology: Challenges for Evidence-Based Medicine and the Physician-Patient Relationship, Ghana Medical Journal, 45(3), 115124 (2011).

Ryff, C., Happiness is Everything, or Is It?, Exploration on the Meaning of Psychological well-Being, Journal of Personality and Social Psychology, 57, 1069-1081 (1989).

Ryff, C., In the Eye of the Beholder: Views of Psychological well-Being in Middle and old-Aged Adults, Psychological and Aging. 4: 195-210 (1989).

Summers, D., Administración de la Calidad, Pearsons Educación, México (2006). 
